\title{
A Generalization of the Spherical Harmonic Addition Theorem
}

\author{
Yasuo Munakata \\ Department of Physics, Kyoto University \\ Received January 12, 1968
}

\begin{abstract}
A generalization of the spherical harmonic addition theorem is proved. The resulting polynomial with four parameters, which corresponds to the Legendre polynomial for the usual spherical harmonic addition theorem, is expressed as four different but equivalent series. Each of them is a finite series of the Gegenbauer polynomials. Thereby the symmetry properties of this polynomial are clarified.
\end{abstract}

\section{$\$ 1$. Introduction and Summary}

In several problems [1] of mathematical physics, there is a need to generalize the spherical harmonic addition theorem. Let us consider the functions of two points $\Omega$ and $\Omega^{\prime}$ on a unit sphere,

$$
B_{L, M}^{l, l_{M}^{\prime}}\left(\Omega, \Omega^{\prime}\right)=\sum_{m} C\left(l l^{\prime} L ; m, M-m\right) Y_{l m}(\Omega) Y_{l^{\prime} M-m}\left(\Omega^{\prime}\right),
$$

which transform by a rotation as the $(2 L+1)$ components of a spherical tensor [2] of rank $L$. In (1), $C\left(l l^{\prime} L ; m, M-m\right)$ is a Clebsh-Gordan coefficient (CGC). From two vectors we can construct only $L+1$ or $L$ linear independent spherical tensors or pseudo tensors of rank $L$. Hence, we can expect and also prove that $B_{L, M}^{l, l^{\prime}}\left(\Omega, \Omega^{\prime}\right)$ can be expanded in the following forms:

$$
\begin{aligned}
& B_{L, M}^{l, l^{\prime}}\left(\Omega, \Omega^{\prime}\right)=\sum_{s=\max \left(0, L_{L}-l^{\prime}\right)}^{\min (l, L)} F_{L ; s}^{l, l^{\prime}}(t) B_{L, M}^{s, L-s}\left(\Omega, \Omega^{\prime}\right) \\
& \text { for } l+l^{\prime}-L=\text { even, }
\end{aligned}
$$

and

In Eqs. (2) and (2'),

$$
B_{L,, M}^{l, l^{\prime}}\left(\Omega, \Omega^{\prime}\right)=\sum_{s=\max \left(1, L-l^{\prime}+1\right)}^{\min (l, L)} F_{L ; s}^{l}, l^{\prime}(t) B_{L,, M}^{s, L-s+1}\left(\Omega, \Omega^{\prime}\right),
$$

$$
t=\cos \Theta=\cos \theta \cos \theta^{\prime}+\sin \theta \sin \theta^{\prime} \cos \left(\varphi-\varphi^{\prime}\right)
$$

with $\Omega=(\theta, \varphi)$ and $\Omega^{\prime}=\left(\theta^{\prime}, \varphi^{\prime}\right)$, and $F_{L ; s}^{l, l^{\prime}}(t)$ is a rotationally invariant function of $t$.

\footnotetext{
${ }^{1} \mathrm{H}$. Joos used functions slightly different from our $B_{L, M}^{s, L-s+1}\left(\Omega, \Omega^{\prime}\right)$ in this case.
} 
The splitting of the two cases for $l+l^{\prime}-L=$ even and odd is due to the space reflexion properties of $B_{L,, M}^{l, l^{\prime}}\left(\Omega, \Omega^{\prime}\right)$;

$$
B_{L, M}^{l, l^{\prime}}\left(-\Omega,-\Omega^{\prime}\right)=(-)^{l+l^{\prime}} B_{L, M}^{l, l_{M}^{\prime}}\left(\Omega, \Omega^{\prime}\right) .
$$

For $L=0(=M), l^{\prime}$ must equal to $l$, hence, Eq. (2) reduces to the well-known spherical harmonic addition theorem, since

$$
F_{0 ; 0}^{l, l}(t)=(-)^{l}(2 l+1)^{1 / 2} P_{l}(t) \text { and } B_{0,0}^{0,0}\left(\Omega, \Omega^{\prime}\right)=1 / 4 \pi \text {. }
$$

Therefore, Eqs. $(2)$ and $\left(2^{\prime}\right)$ just correspond to a generalized addition theorem of the spherical harmonics. Our task is to prove (2) and $\left(2^{\prime}\right)$ and obtain and explicite formula for $F_{L ; s}^{l, l^{\prime}}(t)$.

This problem was first solved by H. Joos [1] in his study of the representation theory of the inhomogeneous Lorenz group and its applications to the scattering amplitudes for two particles with arbitrary spins. Unfortunately his result for $F_{L ; s}^{l, l^{\prime}}(t)$ is rather complicated, a double series in $t$, and its symmetry properties, which will be discussed in $\S 2$ are also obscure.

We meet the same problems as above in the study of a three-particle system (e.g. the quark model for the baryon) and the application of the Regge pole theory to it. This study will be discussed in a separate paper. In this case we must study the analytical properties of $F_{L ; s}^{l, l^{\prime}}(t)$ with respect to $l, l^{\prime}$ and $L$. For this purpose Joos's result is not applicable.

In this paper we shall follow a procedure different from that of Joos, and prove $(2)$ and $\left(2^{\prime}\right)$ in $\S 2$ by using the rotation matrices. The expression for $F_{L: s}^{l, l^{\prime}}(t)$ obtained in $\S 2$ is a single series which contains bilinear products of the Gegenbauer polynomials and the CGC. In $\$ 3$ we simplify this series and obtain four equivalent expressions for $F_{L ; s}^{l, l^{\prime}}(t)$ each of which is a linear combination of the Gegenbauer polynomials. The results in $\S 3$ manifest the symmetry properties of $F_{L ; s}^{l}, l^{\prime}(t)$ and also admit us to study the analytical properties of it with respect to $l, l^{\prime}$ and $L$.

In $\S 4$ the orthogonality relations of $F_{L ; s}^{l, l^{\prime}}(t)$ are discussed. Appendix A is devoted to the proofs of various addition formulas for the bilinear products of the Gegenbauer polynomials. The symmetry properties of $F_{L ; s}^{l} l, l^{\prime}(t)$ are also reestablished by using these addition formulas. In Appendix $\mathrm{B}$, we prove the various mathematical formulas which are used in the text.

\section{$\$ 2$. The Proof of the Addition Theorem}

In this section we shall prove the generalized addition theorems, (2), in the introduction. We follow the procedure which is the most concise one for the derivation of the usual addition theorem of the spherical harmonics [2]. We evaluate (1) in a rotated coordinate system where the point $\Omega^{\prime}$ is on the $z_{1}$-axis and $\Omega$ lies in the $x_{1}-z_{1}$-plane, i.e. $\left(\Omega^{\prime}\right)$ 
$=\left(\theta_{1}^{\prime}=0, \varphi_{1}^{\prime}\right),(\Omega)=\left(\theta_{1}=\Theta, \varphi_{1}=0\right)$ (suffix 1 denotes the coordinate in the rotated coordinate system. In this coordinate system the spherical harmonics take their simple forms,

$$
\begin{aligned}
Y_{l^{\prime} \mu^{\prime}}\left(0, \varphi_{1}^{\prime}\right) & =\delta_{\mu^{\prime}, 0}\left(\left(2 l^{\prime}+1\right) / 4 \pi\right)^{1 / 2}, \\
Y_{l \mu}(\Theta, 0) & =p_{l, \mu}(\cos \Theta)=p_{l, \mu}(t),
\end{aligned}
$$

where $p_{l, m}(t)$ is the normalized associated Legendre function;

$$
p_{l, m}(t)=\frac{1}{2^{l} l !}\left[\frac{2 l+1}{4 \pi} \frac{(l-m) !}{(l+m) !}\right]^{1 / 2}(-\sin \Theta)^{m} \frac{d^{m+l}}{d t^{m+l}}\left(t^{2}-1\right)^{l} .
$$

The relation between $Y_{l m}(\theta, \varphi)$ and $Y_{l_{\mu}}(\Theta, 0)$ on one hand and $Y_{l^{\prime} m^{\prime}}\left(\theta^{\prime}, \varphi^{\prime}\right)$ and $Y_{l^{\prime} \mu^{\prime}}\left(0, \varphi_{1}^{\prime}\right)$ on the other are given in terms of the rotation matrix,

$$
D_{m, m^{\prime}}^{L}(\alpha, \beta, \gamma)=\left(L m\left|e^{-i \alpha L_{z}} e^{-i \beta L_{y}} e^{-i \gamma L_{z}}\right| L m^{\prime}\right),
$$

as follows;

$$
\begin{aligned}
Y_{l m}(\theta, \varphi) & =\sum_{\mu} Y_{l \mu}(\Theta, 0) D_{\mu, m}^{l}\left(-\Phi,-\theta^{\prime},-\varphi^{\prime}\right), \\
Y_{l^{\prime} M-m}\left(\theta^{\prime}, \varphi^{\prime}\right) & =\sum_{\mu} Y_{l^{\prime} \mu^{\prime}}\left(0, \varphi_{1}^{\prime}\right) D_{\mu^{\prime}, M-m}^{l^{\prime}}\left(-\Phi,-\theta^{\prime},-\varphi^{\prime}\right) .
\end{aligned}
$$

In Eq. (6), the azimuthal angle $\Phi$ should be understood as an implicite function of $\theta, \varphi, \theta^{\prime}$ and $\varphi^{\prime}$ which is easily obtained from the ordinary relations between Euler angles. For our purpose the explicite form of $\Phi$ is unnecessary and we drop out the arguments of $D_{m, m^{\prime}}^{L}$ hereafter. Substituting (6) and (3) in (1) we find

$$
B_{L, M}^{l, l^{\prime}}\left(\Omega, \Omega^{\prime}\right)=\left(\left(2 l^{\prime}+1\right) / 4 \pi\right)^{1 / 2} \sum_{\mu} C\left(l l^{\prime} L ; \mu, 0\right) D_{\mu, M}^{L} p_{l, \mu}(t) .
$$

In deriving (7) we have used the relation

$$
D_{\mu, m}^{l} D_{\mu^{\prime}, m^{\prime}}^{l^{\prime}}=\sum_{j} C\left(l l^{\prime} j ; \mu \mu^{\prime}\right) C\left(l l^{\prime} j ; m m^{\prime}\right) D_{\mu+\mu^{\prime}, m+m^{\prime}}^{j}
$$

and the orthogonality relations of the Clebsh-Gordan coefficients. As was stated in the introduction the parity selection rule allows us to treat the two cases, i) $l+l^{\prime}-L=$ even and ii) $l+l^{\prime}-L=$ odd, separately. First we consider the former case.

i) $l+l^{\prime}-L=e v e n$.

In this case, using $C\left(l l^{\prime} L ; \mu, 0\right)=(-)^{l-\mu}\left[(2 L+1) /\left(2 l^{\prime}+1\right)\right]^{1 / 2}$ $C\left(L l l^{\prime} ; \mu,-\mu\right)$ and $p_{l,-\mu}(t)=(-)^{\mu} p_{l, \mu}(t)$, we rewrite (7) as

$$
\begin{aligned}
& (-)^{l}(4 \pi /(2 L+1))^{1 / 2} B_{L, M}^{l, l_{M}^{\prime}}\left(\Omega, \Omega^{\prime}\right)=C\left(L l l^{\prime} ; 00\right) D_{0, M}^{L} p_{l, 0}(t) \\
& \quad+\sum_{\mu=1}^{\min (L, l)} C\left(L l l^{\prime} ; \mu,-\mu\right)(-)^{\mu}\left(D_{\mu, M}^{L}+(-)^{\mu} D_{-\mu, M}^{L}\right) p_{l, \mu}(t) .
\end{aligned}
$$


Setting $l=k$ and $l^{\prime}=L-k(k=0,1,2, \ldots L)$ in $(9)$ we obtain $L+1$ relations $(10)$ :

$$
\begin{gathered}
(-)^{k}(4 \pi /(2 L+1))^{1 / 2} B_{L, M}^{k, L-k}\left(\Omega, \Omega^{\prime}\right)=C(L, k, L-k ; 0,0) D_{0, M}^{L} p_{k, 0}(t) \\
+\sum_{s=1}^{k} C(L, k, L-k ; s,-s)(-)^{s}\left(D_{s, M}^{L}+(-)^{s} D_{-s, M}^{L}\right) p_{k, s}(t) . \\
(k \geqq s, k, s=0,1,2, \ldots, L) .
\end{gathered}
$$

We regard (10) as a linear algebraic equation for $L+1$ unknowns, $D_{0, M}^{L}$ and $\left(D_{s, M}^{L}+(-)^{s} D_{-s, M}^{L}\right)(s=1,2, \ldots, L)$, and try to solve them in terms of $B_{L, M}^{k, L-k}\left(\Omega, \Omega^{\prime}\right)$. Inserting (4) and the explicite form of CGC $C(L, k, L-k ; s,-s)$

$$
=\frac{(-)^{k-s}}{(L-k) !}\left[\frac{(2(L-k)+1) !(2 k) !(L-s) !(L+s) !}{(2 L+1) !(k-s) !(k+s) !}\right]^{1 / 2},
$$

we can rewrite $(10)$, after some manipulations, as

with

$$
X_{k}=\sum_{s=0}^{k} f_{k s}(t) U_{s}
$$

$X_{k}=4 \pi 2^{k} k !(L-k) ![(2 L) ! /(2(L-k)+1) !(2 k+1) !]^{1 / 2} B_{L, M}^{k, L}\left(\Omega, \Omega^{\prime}\right)$, $U_{0}=D_{0, M}^{L}$,

$U_{s}=((L+s) !(L-s) !)^{1 / 2}(-\sin \Theta)^{s}\left(D_{s, M}^{L}+(-)^{s} D_{-s, M}^{L}\right) \quad(s=1,2, \ldots L)$ and

$$
f_{k s}(t)=\frac{1}{(k+s) !} \frac{d^{k+s}}{d t^{k+s}}\left(t^{2}-1\right)^{k}=\frac{2^{k-s}(2 s) ! k !}{(k+s) ! s !} C_{k-\delta}^{\frac{1}{2}+s}(t),
$$

where $C_{n}^{v}(t)$ is a Gegenbauer polynomial [3].

We prove in the Appendix A a relation

with

$$
\sum_{s=p}^{k} f_{k s}(t)(-)^{s-p} h_{s p}(t)=\delta_{k p}
$$

$$
\begin{aligned}
h_{k s}(t) & =\frac{k}{s} \frac{1}{(k-s) !}\left(1-t^{2}\right)^{k} \frac{d^{k-s}}{d t^{k-s}}\left(1-t^{2}\right)^{-s} \\
& =\frac{(-2)^{k-s} k !(k+s-1) !}{s !(2 k-1) !} C_{k-s}^{\frac{1}{2}-k}(t), \quad(k \geqq s) .
\end{aligned}
$$

By the use of (14), we can solve (12) as

$$
U_{k}=\sum_{s=0}^{k} h_{k s}(t) X_{s}(-)^{k-s} .
$$

\footnotetext{
${ }^{2}$ For $k=s=0$, we define $h_{00}(t)=1$.
} 
Inserting (16) and (13) in (9) we get finally

with

$$
B_{L, M}^{l, l^{\prime}}\left(\Omega, \Omega^{\prime}\right)=\sum_{s=0}^{\min (l, L)} F_{L: s}^{l, l^{\prime}}(t) B_{L, M}^{s, L-s}\left(\Omega, \Omega^{\prime}\right),
$$

$$
\begin{aligned}
F_{L ; s}^{l, l^{\prime}}(t)= & (-)^{l}(L-s) !\left[\frac{(2 l+1)(2 L+1) !}{(2(L-s)+1) !(2 s+1) !}\right]^{1 / 2} \\
& \cdot \sum_{k=s}^{\min (l, L)}\left[\frac{(l-k) !}{(L-k) !(L+k) !(l+k) !}\right]^{1 / 2} \\
& \cdot(-)^{k} 2 k(k+s-1) ! C\left(L l l^{\prime} ; k,-k\right) C_{l-k}^{\frac{1}{2}+k}(t) C_{k-8}^{\frac{1}{2}-k}(t) .
\end{aligned}
$$

At this stage we are reminded the symmetry properties of $B_{L, M} l, l^{\prime}\left(\Omega, \Omega^{\prime}\right)$. Since $C\left(l l^{\prime} L ; m, M-m\right)=C\left(l^{\prime} l L ; M-m, m\right)(-)^{l+l^{\prime}-L}$, the defining equation of $B_{L, M}, l_{M}^{\prime}\left(\Omega, \Omega^{\prime}\right)$ leads to

$$
B_{L, M}^{l, l^{\prime}}\left(\Omega, \Omega^{\prime}\right)=(-)^{l+l^{\prime}-L} B_{L, M}^{l, l^{\prime}}\left(\Omega^{\prime}, \Omega\right) .
$$

By applying (19) in our case $\left(l+l^{\prime}-L=\right.$ even) we learn from (17)

$$
B_{L, M}^{l, l^{\prime}}\left(\Omega, \Omega^{\prime}\right)=\sum_{s=0}^{\min (l, L)} F_{L ; s}^{l, l^{\prime}}(t) B_{L, M}^{s, L-s}\left(\Omega, \Omega^{\prime}\right)
$$

$=\sum_{s=0}^{\min \left(l^{\prime}, L\right)} F_{L ; s}^{l^{\prime}, l}(t) B_{L, M}^{s, L-s}\left(\Omega^{\prime}, \Omega\right)=\sum_{s=\max \left(0, L-l^{\prime}\right)}^{L} F_{L ; L-s}^{l^{\prime}, l}(t) B_{L, L}^{s, L-s}\left(\Omega, \Omega^{\prime}\right)$,

where we have changed summation variable $s$ to $L-s$ and again used (19) in the last line. Eq. (20) holds identically in the four independent variables $\theta^{\prime}, \varphi^{\prime}, \Omega$ and $t$, and $L+1$ functions $B_{L, M}^{s, L-s}\left(\Omega, \Omega^{\prime}\right)$ $(s=0,1,2, \ldots L)$ are independent, we are lead to the symmetry relations for $F_{L ; s}^{l, l^{\prime}}(t)$;

and

$$
F_{L ; s}^{l, l^{\prime}}(t)=F_{L ; L-s}^{l^{\prime}, l}(t), \quad(s==0,1,2, \ldots, L)
$$

$$
\begin{aligned}
F_{L ; s}^{l, l^{\prime}}(t) & =0, \text { for } s=0,1, \ldots, L-l^{\prime}-1, \text { if } L>l^{\prime}, \\
\left(l+l^{\prime}-L\right. & =\text { even }) .
\end{aligned}
$$

From (20) and (21) we get (2) in $\$ 1$ for the case $l+l^{\prime}-L=$ even.

Returning to $\mathrm{Eq}_{\mathrm{q}}$. (18) we find that $F_{L l ; s}^{l, l^{\prime}(t)}$ is a polynomial in $t$ of degree $n$, where

with

$$
n=\min . \text { of }\left(l-s, l^{\prime}-L+s\right) \text {, }
$$

$$
\max \left(0, L,-l^{\prime}\right) \leqq s \leqq \min (L, l),
$$

since $C_{m}^{v}(t)$ is a polynomial of degree $m$. In Fig. 1 we show the contour lines for the degree of the polynomials $F_{L ; s}^{l, l^{\prime}}(t)$ for given fixed values of $l, l^{\prime}$. 


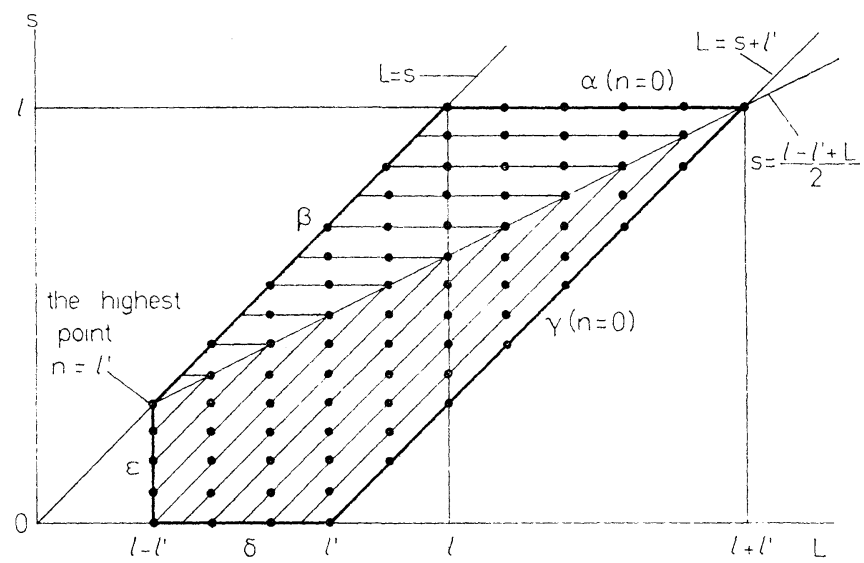

Fig. 1. The contour lines for the degree $n$ of the polynomials $F_{L ; s}^{l, l^{\prime}}(t)$ in the $L-s$ plane for fixed $l$ and $l^{\prime}$. We draw for a typical case $2 l^{\prime}>l>l^{\prime}$ and $l+l^{\prime}-L$ $=$ even. The dots on this diagram denote the permissible points

ii) $l+l^{\prime}-L=o d d$

In this case, as $C\left(L l l^{\prime} ; 00\right)=0$, the corresponding equation to (12) contains $L$ unknowns instead of $L+1$. Taking into account this minor change and the phase relations of CGC, we can proceed the almost similar calculations as those in $\mathrm{i}$ ), hence, we write the final results only:

with

$$
B_{L, M}^{l, l^{\prime}}\left(\Omega, \Omega^{\prime}\right)=\sum_{s=\max \left(1, L-l^{\prime}+1\right)}^{\min (l, L)} F_{L ; s}^{l, l^{\prime}}(t) B_{L, M}^{s, I,-s+1}\left(\Omega, \Omega^{\prime}\right),
$$

$F_{l i: s}^{l, l^{\prime}}(t)=(-)^{l}(L-s) !\left[\frac{(2 l+1)(2 L+2) !}{(2(L-s)+3)(2 s+1)(2(L-s)+1) !(2 s-1) !}\right]^{1 / 2}$

$\cdot \sum_{k=s}^{\min (l, L)}\left[\frac{(l-k) !}{(L-k) !(L+k) !(l+k) !}\right]^{1 / 2}(-)^{k}(k+s-1) ! C\left(L l l^{\prime} ; k,-k\right)$

$\cdot C_{l-k}^{\frac{1}{2}+k}(t) C_{k-s}^{\frac{1}{2}-k}(t)$.

The symmetry relations of $F_{L ; s}^{l, l^{\prime}}(t)$ in this case are

and

$$
F_{L ; s}^{l, l^{\prime}}(t)=F_{L ; L-s+1}^{l^{\prime}, l}(t), \quad(s=1,2, \ldots, L)
$$

$$
F_{L ; s}^{l, l^{\prime}}(t)=0, \text { for } s=1,2, \ldots, L-l^{\prime}, \quad \text { if } L>l^{\prime} .
$$

The degree $n$ of the polynomial $F_{l, ; s}^{l, l^{\prime}}(t)$ is given by

with

$$
n=\min . \text { of }\left(l-s, l^{\prime}-L+s-1\right) \text {, }
$$

$$
\max \left(1, L-l^{\prime}\right) \leqq s \leqq \min (L, l)
$$




\section{\$3. Evaluations and Rewritings of $\boldsymbol{F}_{\boldsymbol{L} ; \boldsymbol{s}}^{\boldsymbol{l}, \boldsymbol{l}^{\prime}}(\boldsymbol{t})$}

The expressions for $F_{L ; s}^{l, l^{\prime}}(t)$ obtained in $\S 2$, Eqs. (18) and $\left(18^{\prime}\right)$, are rather complicated and the symmetry relations (21) and (21') are also obscure. In this section we proceed further calculations to simplify (18) and $\left(18^{\prime}\right)$. For this purpose, it is necessary to treat the two cases i) $l+l^{\prime}-L=$ even and ii) $l+l^{\prime}-L=$ odd separately.

i) $l+l^{\prime}-L=$ even

Starting from (18) we insert the explicite form of the CGC given by RACAH [4] in it,

$$
\begin{aligned}
& C\left(L l l^{\prime} ; k,-k\right)=(-)^{L-k} l^{\prime} !\left[\left(2 l^{\prime}+1\right)\left(L+l^{\prime}-l\right) !\left(l+L-l^{\prime}\right) !\right. \\
& \left.\cdot\left(l+l^{\prime}-L\right) !(L+k) !(L-k) !(l+k) !(l-k) !\left(\left(L+l+l^{\prime}+1\right) !\right)^{-1}\right]^{1 / 2} \\
& \cdot \sum_{v} \frac{(-)^{\prime}}{v !\left(L+l^{\prime}-l-v\right) !(L-k-v) !\left(l^{\prime}-v\right) !\left(l-l^{\prime}+k+v\right) !(l-L+v) !} .
\end{aligned}
$$

Apart from a common factor we must calculate the following double series of the bi-linear products of Gegenbauer polynomials:

$$
\begin{aligned}
& I=\sum_{k}^{\min (l, L)} \sum_{v} \frac{(-)^{v} 2 k(k+s-1) !(l-k) !}{v !(2 b-v) !(l-L+2 b-v) !(l-L+v) !} \\
& \cdot C_{l-k}^{\frac{1}{2}+k}(t) C_{k-s}^{\frac{1}{2}-k}(t) \\
& \cdot(L-k-v) !(L-2 b+k+v) !
\end{aligned}
$$

with $b=\left(L+l^{\prime}-l\right) / 2,(b$ : non negative integer).

In Eqs. (23) and (24), the integral index $v$ assumes all integral values such that none of the factorial arguments are negative. In order to treat the various cases, originated from the relative order of magnitudes of $l, l^{\prime}, L$ and $s$, in a unified way, we make a following convention:

The range of the summations over $\boldsymbol{v}$ and $k$ are enlarged formally to cover the various cases simultaneously, thereby the terms which include negative integral factorial arguments in the denominator or negative integral index $m$ of $C_{m}^{\nu}(t)$ are put equal to zero. (a).

Under this convention (a) the upper and the lower limits of the summation variables may be dropped. We note that the convention (a) is a natural one because $1 / m !=1 / \Gamma(m+1)=0$, for $m=-1,-2, \ldots$, and $C_{m}^{v}(t)=0$ are consistent with their definition by the generating function

$$
\left(1-2 x t+x^{2}\right)^{-v}=\sum_{m=0}^{\infty} C_{m}^{v}(t) x^{m} .
$$

In order to simplify the Eq. (24), the following addition formula for the bilinear products of the Gegenbauer polynomials is useful,

$$
\begin{aligned}
& \sum_{k} \frac{2 k(l-k) !(k+s-1) !}{(n-k) !(n+k) !} C_{l-k}^{\frac{1}{2}+k}(t) C_{k-s}^{\frac{1}{2}-k}(t) \\
& \quad=\frac{(-)^{n-s}(l-n) !}{(n-s) !} C_{l-2 n+s}^{\frac{1}{2}+n}(t), \quad \text { for } \quad l \geqq n,
\end{aligned}
$$


the proof of which is given in the Appendix A. In order to apply Eq. (26) to (24), the double sum in (24) is written as $\sum_{k} \sum_{v}=\sum_{\nu=0}^{2 b} \sum_{k=s}^{L-v}$ and the terms $v=b \pm m(1 \leqq m \leqq b)$ are combined,

$$
\begin{aligned}
I= & \frac{(-)^{b}}{(b ! c !)^{2}} \sum_{k=s}^{a} 2 k(k+s-1) !(l-k) ! f_{k}^{a} C_{l-k}^{\frac{1}{2}+k}(t) C_{k-s}^{\frac{1}{2}-k}(t) \\
& +\sum_{m=1}^{b} \frac{(-)^{b+m}}{(b+m) !(b-m) !(c+m) !(c-m) !} \\
& \cdot \sum_{k=s}^{a+m} 2 k(k+s-1) !(l-k) ! g_{m ; k}^{a} C_{l-k}^{\frac{1}{2}+k}(t) C_{k-s}^{\frac{1}{2}-k}(t),
\end{aligned}
$$

where we have the abbreviations

$$
\begin{array}{rlrl}
a & =\left(L+l-l^{\prime}\right) / 2, & L & =a+b, \\
b & =\left(L+l^{\prime}-l\right) / 2, \quad \text { or } \quad l^{\prime} & =b+c, \\
c & =\left(l+l^{\prime}-L\right) / 2, & l & =c+a, \quad\left(L+l-l^{\prime}=\text { even }\right)
\end{array}
$$

and

$$
\begin{aligned}
f_{k}^{a} & =\frac{1}{(a-k) !(a+k) !}, \\
g_{i n ; k}^{a} & =\frac{1}{(a-k-m) !(a+k+m) !}+\frac{1}{(a-k+m) !(a+k-m) !} .
\end{aligned}
$$

Now we substitute in (27)

$$
g_{m ; k}^{a}=\frac{2 m}{(2 a) !} \sum_{\sigma=0}^{b} \frac{(-)^{\sigma}(2 m-\sigma-1) !(2(a+m-\sigma)) !}{\sigma !(2(m-\sigma)) !} f_{k}^{a}+m-\sigma, \text { for } m \geqq 1,
$$

and

$$
g_{0 ; k}^{a}=2 f_{k}^{a},
$$

which are proved in the Appendix B. Performing the sum over $k$ by using (26) we get

Putting

$$
\begin{aligned}
I= & \frac{(-)^{L-s}}{(a-s) !} C_{c-a+s}^{\frac{1}{2}+a}(t)\left[(c !)^{-1}(b !)^{-2}\right. \\
& \left.+2 c ! \sum_{m=1}^{b}((b+m) !(b-m) !(c+m) !(c-m) !)^{-1}\right] \\
& +\frac{(-)^{L-s}}{(2 a) !} \sum_{\varrho=1}^{b} \frac{(c-\varrho) !(2(a+\varrho)) !}{(2 \varrho) !(a+\varrho-s) !} C_{c-a+s-2 \varrho}^{\frac{1}{2}+a+\varrho}(t) \\
& \cdot \sum_{m=\varrho}^{b} \frac{2 m(m+\varrho-1) !}{(m-\varrho) !(b+m) !(b-m) !(c+m) !(c-m) !} .
\end{aligned}
$$

$$
\varphi_{b, c}^{\varrho}=\sum_{m=\varrho}^{b} \frac{2 m(m+\varrho-1) !}{(m-\varrho) !(b+m) !(b-m) !(c+m) !(c-m) !},
$$


we write the square bracket in the first term in (31) as $c ! \psi_{b}, c$ with

$$
\psi_{b, c}=\varphi_{b, c}^{0}-(b ! c !)^{-2} .
$$

Performing the sum over $m$ in (32) and (33) in the Appendix B we get $q_{h, c}^{\varrho}=\frac{(2 \varrho) ! b ! c !(2(b+c-\varrho)) !}{\varrho !(b-\varrho) !(c-\varrho) !(b+c) !(b+c-\varrho) !(2 b) !(2 c) !}$, for $\varrho \geqq 1$, and

$$
\psi_{l, c}=\frac{(2(b+c)) !}{(2 b) !(2 c) !((b+c) !)^{2}} .
$$

Collecting the results (31), (34) and (35), remembering (23), (18) and the convention (a) we obtain the final result,

with

$$
\begin{aligned}
F_{L: s}^{l, l^{\prime}}(t)= & (L-s) ! c ! N_{L: ;}^{l, l^{\prime}} \sum_{\varrho=\min (b,[(c-a+s) / 2])}^{\min (0, s-a)}\left(\begin{array}{l}
b \\
\varrho
\end{array}\right) \\
& \cdot \frac{(2(b+c-\varrho)) !(2(a+\varrho)) !}{(b+c-\varrho) !(a+\varrho-s) !} C_{c-a \div s-2,}^{\frac{1}{2}+a+\varrho}(t),
\end{aligned}
$$

$$
\begin{aligned}
& N_{L ; s}^{l, l^{\prime}}=(-)^{l-s}\left[\frac{(2 l+1)\left(2 l^{\prime}+1\right)}{(2 s+1) !(2(L-s)+1) !\left(L+l-l^{\prime}\right) !}\right. \\
& \left.\frac{\cdot(2 L+1) !}{\cdot\left(L+l^{\prime}-l\right) !\left(l+l^{\prime}-L\right) !\left(L+l+l^{\prime}+1\right) !}\right]^{1 / 2} \\
& =(-)^{c+a-s}\left[\frac{(2(c+a)+1)(2(b+c)+1)}{(2 s+1) !(2(a+b-s)+1) !}\right. \\
& \left.\frac{\cdot(2(a+b)+1) !}{\cdot(2(a+b+c)+1) !(2 a) !(2 b) !(2 c) !}\right]^{1 / 2} \text {. }
\end{aligned}
$$

By using the symmetry properties of $F_{L: s}^{l, l^{\prime}}(t)$, Eq. (21), we obtain the second expression for $F_{L ; s}^{l, l^{\prime}}(t)$;

$$
\begin{aligned}
& F_{L ; s}^{l, l^{\prime}}(t)=s ! c ! N_{L ; ; s}^{l, l^{\prime}} \\
& \min _{\varrho=\max (a,[(c+a-s) / 2])} \sum^{\urcorner}\left(\begin{array}{l}
a \\
\varrho
\end{array}\right) \frac{(2(a+s)}{(a+c-\varrho) !(s-a+\varrho) !} C_{a+c-s-2 \varrho}^{\frac{1}{2}+b+\varrho}(t) .
\end{aligned}
$$

Furthermore, we shall show in the following, by using the recurrence relations for $C_{n}^{\nu}(t)$, that $F_{L ; s}^{l, l^{\prime}}(t)$ can be written in the third and the fourth forms. Repeated use of the recurrence relation ${ }^{4}$

leads to

$$
v C_{n-2}^{v+1}(t)=v C_{n}^{v+1}(t)-(n+v) C_{n}^{v}(t)
$$

$$
\begin{aligned}
C_{c-a+s-2 \varrho}^{\frac{1}{2}+a+\varrho}(t)= & \frac{\varrho !(a+\varrho) !(2(c+s+1)) !}{(2(a+\varrho)) !(c+s+1) !} \\
& \cdot \sum_{\nu=0}^{\varrho} \frac{(-)^{\varrho-v}(2(a+v)) !(c+s+1-\varrho+v) !}{(\varrho-v) ! v !(a+v) !(2(c+s+1-\varrho+v)) !} C_{c-a+s}^{\frac{1}{2}+a+v}(l),
\end{aligned}
$$

${ }^{3}[a / 2]$ means the maximum integer which does not exceed $a / 2$.

4 See the Appendix A, (A.4). 
the validity of which is ascertained by the mathematical induction about $\varrho$. Substituting (39) into (36), remembering the convention (a), we interchange the order of the summations over $\nu$ and $\varrho$ and rewrite (36) as

$$
\begin{aligned}
F_{L ; s}^{l, l^{\prime}}(t) & =(L-s) ! b ! c ! N_{L ; s}^{l, l^{\prime}} \frac{(2(c+s+1)) !}{(c+s+1) !} \\
& \cdot \sum_{v=0}^{b} \frac{(2(a+v)) !}{v !(a+v) !} C_{c-a+s}^{1}+a+v \\
& \cdot \sum_{\varrho=v}^{b} \frac{(-)^{Q-v}(a+\varrho) !(2(b+c-\varrho)) !(c+s+1-\varrho+v) !}{(\varrho-v) !(a+\varrho-s) !(b+c-\varrho) !(b-\varrho) !(2(c+s+1-\varrho+v)) !} .
\end{aligned}
$$

The last factor in $(40)$, the series over $\varrho$, can be written with the change of summation variable $\varrho \rightarrow \tau=\varrho-\nu$ as

$\phi_{c, a}^{b-v}+b, s$

$=\sum_{\tau=0}^{b-v} \frac{(-)^{\tau}(a+v+\tau) !(2(b+c-v-\tau)) !(c+s+1-\tau) !}{\tau !(a+v+\tau-s) !(b+c-v-\tau) !(b-v-\tau) !(2(c+s+1-\tau)) !}$.

(41) is evaluated in the Appendix B as

$\phi_{c, a}^{b-v}+b, s$

$=\frac{(-)^{b-v}(c+s+1) !(2 c) !(2(a+b+c)+1) ! s !(a+v) !(a+c+v) !}{(2(c+s+1)) ! c !(a+b+c) !(a+b-s) !(b-v) !(s-b+v) !(2(a+c+v)+1) !}$.

Substituting (42) into (40) we obtain the third expression for $F_{L ; s}^{l, l^{\prime}}(t)$,

$F_{L ; s}^{l, l^{\prime}}(t)=s !(2 c) ! N_{L ; s}^{l, l^{\prime}} \sum_{\varrho=0}^{\min (b, s)}\left(\begin{array}{l}b \\ \varrho\end{array}\right) \frac{(-)^{\varrho}(2(L-\varrho)) !(2(d-\varrho))_{2} \varrho}{(s-\varrho) !(d-\varrho)_{\varrho}} C_{l^{\prime}-L+s}^{\frac{1}{2}+L-\varrho}(t)$, with $d=a+b+c+1=\left(l+l^{\prime}+L\right) / 2+1$.

Symmetry properties of $F_{L ; s}^{l, l^{\prime}}(t)$ lead again from (43) to $F_{L ; s}^{l, l^{\prime}}(t)$

$$
=(L-s) !(2 c) ! N_{L ; s}^{l, l^{\prime}} \sum_{\varrho=0}^{\min (a, L-s)}\left(\begin{array}{l}
a \\
\varrho
\end{array}\right) \frac{(-)^{\varrho}(2(L-\varrho)) !(2(d-\varrho))_{2 \varrho}}{(L-s-\varrho) !(d-\varrho)} C_{l-s}^{\frac{1}{2}+L-\varrho}(t) \text {. }
$$

Thus we have obtained four equivalent expressions for $F_{L ; s}^{l, l^{\prime}}(t),(36)$, (38), (43) and (44). On the boundary curves $\alpha, \beta, \gamma, \delta$ and $\varepsilon$ in Fig. 1, $F_{L ; s}^{l, l^{\prime}}(t)$ consists of single term. We list them in the above order by using the Eqs. (36) or (38), (36) or (44), (36) or (38), (38) or (43) and (36) or (43), respectively;

$$
\begin{aligned}
F_{L ; l}^{l, l^{\prime}}(t) & =(2 l) !\left(L+l^{\prime}-l\right) ! N_{L ; l}^{l, l^{\prime},} \text { on } \alpha, \\
F_{L ; L}^{l, l^{\prime}}(t) & =(2 L) !\left(l+l^{\prime}-L\right) ! N_{L ; l}^{l, l^{\prime}} C_{l-L}^{\frac{1}{2}+L}(t), \quad \text { on } \beta, \\
F_{L ; L-l^{\prime}}^{l, l^{\prime}}(t) & =\left(2 l^{\prime}\right) !\left(l+l^{\prime}-L\right) ! N_{L ; L-l^{\prime}}^{l, l^{\prime}}, \quad \text { on } \gamma, \\
F_{L^{\prime} ; 0}^{l, l^{\prime}}(t) & =\left(l+l^{\prime}-L\right) !(2 L) ! N_{L ; 0}^{l, l^{\prime}} C_{l^{\prime}-L}^{\frac{1}{2}+L}(t), \quad \text { on } \delta, \\
F_{l-l^{\prime} ; s}^{l, l^{\prime}}(t) & =\left(2 l^{\prime}\right) !\left(2\left(l-l^{\prime}\right)\right) ! N_{l-l^{\prime} ; s}^{l, l^{\prime}} C_{l^{\prime}-L+s}^{\frac{1}{2}+l-l^{\prime}}(t), \quad \text { on } \varepsilon .
\end{aligned}
$$

${ }^{5}(a)_{n}=(a+n-1) ! /(a-1) !$ 
For general values $\left(l, l^{\prime}, L\right.$ and $\left.s\right)$ we can choose in a same way as above the simplest expression from these four equivalent ones.

ii) $l+l^{\prime}-L=$ odd

We start from $\left(18^{\prime}\right)$ in $\S 2$ and substitute (23) in it. Apart from a common factor we have to evaluate

$$
\begin{aligned}
& J=\sum_{k=s}^{\min (l, L)} \sum_{v} \frac{(-)^{v}(k+s-1) !(l-k) !}{v !\left(L+l^{\prime}-l-v\right) !\left(l^{\prime}-v\right) !(l-L+v) !} \\
& \frac{\cdot C_{k-s}^{\frac{1}{2}+k}(t) C_{k-s}^{\frac{1}{2}-k}(t)}{\cdot(L-k-v) !\left(l-l^{\prime}+k+v\right) !},
\end{aligned}
$$

instead of $I,(24)$. We set in this case

$$
\begin{array}{ll}
a=\left(L+l-l^{\prime}-1\right) / 2, & L=a+b+1, \\
b=\left(L+l^{\prime}-l-1\right) / 2, & \text { or } \quad l^{\prime}=b+c+1, \\
c=\left(l+l^{\prime}-L-1\right) / 2, & l=c+a+1 . \\
d=a+b+c+2=\left(L+l+l^{\prime}+1\right) / 2,
\end{array}
$$

We follow the convention (a) and write the double sum as $\sum_{k} \sum_{v}=\sum_{\nu=0}^{2 b+1} \sum_{k}^{1}$, combine the terms $v=b-m$ and $v=b+1+m$, we have

with

$$
\begin{aligned}
J= & \sum_{m=0}^{b} \frac{(-)^{b-m}}{(b-m) !(b+1+m) !(c-m) !(c+1+m) !} \\
& \cdot \sum_{k} 2 k(k+s-1) !(l-k) ! h_{m ; k}^{a+1} C_{l-k}^{\frac{1}{2}+k}(t) C_{k-s}^{\frac{1}{2}-k}(t),
\end{aligned}
$$

$$
\begin{aligned}
2 k h_{m ; k}^{a+1}= & \frac{1}{(a+m+1-k) !(a-m+k) !} \\
& -\frac{1}{(a+m+1+k) !(a-m-k) !} .
\end{aligned}
$$

We prove in the Appendix B that

$$
h_{m ; k}^{a+1}=\sum_{\varrho=0}^{m} \frac{(-)^{\sigma}(2 m-\sigma) !(2(a+m-\sigma)+1) !}{\sigma !(2(m-\sigma)) !(2 a+1) !} f_{k}^{a+m-\sigma+1,}
$$

with $f_{k}^{a}$ given by (29). Substituting (50) into (48), we apply (26) to get

$$
J=\frac{(-)^{a+b+1-s}}{(2 a+1) !} \sum_{\varrho=0}^{b} \frac{(c-\varrho) !(2(a+\varrho)+1) !}{(a+\varrho-s+1) !(2 \varrho) !} \chi_{b, c}^{\varrho} C_{c-a-1+s-2 \varrho}^{\frac{1}{2}+a+1+\varrho}(t),
$$

with

$$
\chi_{b, c}^{\varrho}=\sum_{m=\varrho}^{b} \frac{(m+\varrho) !}{(b-m) !(b+m+1) !(c-m) !(c+m+1) !(m-\varrho) !} .
$$


We evaluate $\chi_{b, c}^{\varrho}$ in the Appendix B as

$\chi_{b, c}^{\varrho}=\frac{(2 \varrho) ! b ! c !(2(b+c-\varrho)+1) !}{\varrho !(b-\varrho) !(c-\varrho) !(2 b+1) !(2 c+1) !(b+c-\varrho) !(b+c+1) !}$.

Collecting the results (51) and (53), remembering (23), we get from (18') the final result

$$
\begin{aligned}
& F_{L ; s}^{l, l^{\prime}}(t)=(L-s) ! c ! N_{L ; s}^{l, l^{\prime}} \\
& \min (b,[(c-a+s-1) / 2]) \\
& \cdot \sum_{\varrho=\max (0, s-a-1)}^{b}\left(\begin{array}{l}
b \\
\varrho
\end{array}\right) \frac{(2(b+c-\varrho)+1) !(2(a+\varrho)+1) !}{(b+c-\varrho) !(a+1-s+\varrho) !} C_{c-a-1+s-2 \varrho}^{\frac{1}{2}+a+1+\varrho}(t),
\end{aligned}
$$

with

$$
\begin{aligned}
N_{L^{\prime} ; s}^{l, l^{\prime}}= & (-)^{l-s}\left[\frac{(2 l+1)\left(2 l^{\prime}+1\right)(2(L-s+1))}{(2(L-s)+3) !(2 s+1) !}\right. \\
& \left.\cdot \frac{(2 s)(2(L+1)) !}{\left(l+l^{\prime}+L+1\right) !\left(l+l^{\prime}-L\right) !\left(l+L-l^{\prime}\right) !\left(l^{\prime}+L-l\right) !}\right]^{1 / 2} \\
= & (-)^{c+a-s-1}\left[\frac{(2(c+a)+3)(2(b+c)+3)}{(2 s+1) !(2(a+b-s)+5) !}\right. \\
& \cdot(2 s)(2(a+b-s)+3)(2(a+b+2)) ! \\
& \cdot(2 d) !(2 a+1) !(2 b+1) !(2 c+1) !
\end{aligned}
$$

We apply the symmetry properties of $F_{L ; 8}^{l, l^{\prime}}(t)$, Eq. $\left(21^{\prime}\right)$ in $\S 2$, to (54) and have the second expression for $F_{L ; s}^{l, l^{\prime}}(t)$ :

$$
\begin{aligned}
F_{L ; s}^{l, l^{\prime}}(t)= & (s-1) ! c ! N_{L ; s}^{l, l^{\prime}} \sum_{\varrho=\max (0, a-s+1)}^{\min (a,[(c+a-s+1) / 2])}\left(\begin{array}{l}
a \\
\varrho
\end{array}\right) \\
& \cdot \frac{(2(a+c-\varrho)+1) !(2(b+\varrho)+1) !}{(a+c-\varrho) !(s-1-a+\varrho) !} C_{c+a-s+1-2 \varrho}^{\frac{1}{2}+b+1+\varrho}(t) .
\end{aligned}
$$

The third and the fourth expressions for $F_{L ; s}^{l, l^{\prime}}(t)$ are obtained by the similar procedures as those in i). We insert (39) with $a \rightarrow a+1$ into (54), change the order of summations over $v$ and $\varrho$. The relevant formula corresponding to $(42)$ is

$$
\begin{aligned}
\psi_{c, a}^{b-v}+b+1, s \equiv & \sum_{\tau=0}^{b-v} \frac{(-)^{\tau}(a+v+\tau) !(2(b+c-v-\tau)+1) !}{\tau !(a+v+\tau-s+1) !(b+c-v-\tau) !} \\
& \frac{\cdot(c+s+1-\tau) !}{\cdot(b-v-\tau) !(2(c+s+1-\tau)) !} \\
= & \frac{(-)^{b-v}(s-1) !(a+v) !(2(a+b+c)+3) !}{(a+b-s+1) !(b-v) !(a+b+c+1) !} \\
& \frac{\cdot(2 c+1) !(c+s+1) !(a+c+v+1) !}{\cdot(2(c+s+1)) !(2(a+c+v)+3) !(s-b+v-1) ! c !},
\end{aligned}
$$

the proof of which is given in the Appendix B. The third expression for $F_{L ; s}^{l, l^{\prime}}(t)$ in this case is thus given by

$$
\begin{aligned}
F_{L ; s}^{l, l^{\prime}}(t)= & (s-1) !(2 c+1) ! N_{L ; s}^{l, l^{\prime}} \sum_{\varrho=0}^{\min (b, s-1)}\left(\begin{array}{l}
b \\
\varrho
\end{array}\right) \\
& \cdot \frac{(-)^{\varrho}(2(L-\varrho)-1) !(2(d-\varrho))_{2} \varrho}{(s-\varrho-1) !(d-\varrho)_{\varrho}} C_{l^{\prime}-L-1+s}^{\frac{1}{2}+L-\varrho}(t) .
\end{aligned}
$$


The fourth one obtained by $\left(21^{\prime}\right)$ is given by

$$
\begin{aligned}
F_{L ; s}^{l, l^{\prime}}(t)= & (L-s) !(2 c+1) ! N_{L ; ; s}^{l, l^{\prime}} \sum_{\varrho=0}^{\min (a, s-1)}\left(\begin{array}{l}
a \\
\varrho
\end{array}\right) \\
& \cdot \frac{(-)^{\varrho}(2(L-\varrho)-1) !(2(d-\varrho))_{2}}{(L-s-\varrho) !(d-\varrho)_{\varrho}} C_{l-s}^{\frac{1}{2}+L-\varrho}(t) .
\end{aligned}
$$

Notice that in these formulas $a, b, c$ and $d$ are defined by (47) and $N_{L ; s}^{l, l^{\prime}}$ by (55).

Finally we remark that if we try to study the analytic properties of $F_{L ; s}^{l, l^{\prime}}(t)$ with respect to $l, l^{\prime}$ and $L$ the formulas obtained in this section are appropriate, since the analytical properties of $C_{n}^{v}(t)$ in $n$ and $v$ are studied by several authors [5].

\section{\$ 4. Orthogonality Relations for $F_{L ; s}^{l, l^{\prime}}(t)$}

In this section we shall study the orthogonality relations for $F_{L ; s}^{l, l^{\prime}}(t)$. We first note that $B_{L, M}^{l, l^{\prime}}\left(\Omega, \Omega^{\prime}\right)$ as defined by $(1)$ is a spherical tensor of rank $L$, hence the $\operatorname{sum} \sum_{M} B_{L, M_{M}}^{l, l^{\prime}}\left(\Omega, \Omega^{\prime}\right) B_{L, M}^{l^{\prime \prime}, l^{\prime \prime \prime}{ }^{*}}\left(\Omega, \Omega^{\prime}\right)$ is a rotational invariant and depends only on $t$. Indeed from (7) in $\S 2$ we get

$$
\begin{gathered}
\sum_{M} B_{L, M}^{l, l^{\prime}}\left(\Omega, \Omega^{\prime}\right) B_{L, M^{\prime \prime}, l^{\prime \prime \prime}}\left(\Omega, \Omega^{\prime}\right)=(2 L+1) / 4 \pi \cdot(-)^{l+l^{\prime \prime}} \\
\cdot \sum_{k=-n}^{n} C\left(L l l^{\prime} ; k,-k\right) C\left(L l^{\prime \prime} l^{\prime \prime \prime} ; k,-k\right) p_{l, k}(t) p_{l^{\prime \prime}, k}(t),
\end{gathered}
$$

with $n=\min \left(l, l^{\prime \prime}, L\right)$, where we have used the unitarity of $D_{m, M}^{L}$. Integrating (61) over $t$, we get

$$
\int_{-1}^{1} d t \sum_{M} B_{L, M}^{l, l^{\prime}}\left(\Omega, \Omega^{\prime}\right) B_{L, M}^{l^{\prime \prime}, l^{\prime \prime \prime} *}\left(\Omega, \Omega^{\prime}\right)=(2 L+1) / 8 \pi^{2} \cdot \delta_{l l^{\prime \prime}} \delta_{l^{\prime} l^{\prime \prime \prime}} .
$$

In the following we treat separately the cases $l+l^{\prime}-L=$ even and odd:

i) $l+l^{\prime}-L=$ even

We substitute (2) into (62) and defining

we have

$$
G_{L}^{s, s^{\prime}}(t)=(4 \pi)^{2} \sum_{M} B_{L, M}^{s, L-s}\left(\Omega, \Omega^{\prime}\right) B_{L, M}^{s^{\prime}, L^{-s^{\prime *}}}\left(\Omega, \Omega^{\prime}\right)
$$

$$
\sum_{s, s^{\prime}} \int_{-1}^{1} d t F_{L ; s}^{l, l^{\prime}}(t) G_{L}^{s, s^{\prime}}(t) F_{L ; s^{\prime}}^{l^{\prime \prime}, l^{\prime \prime \prime}}(t)=2(2 L+1) \delta_{l l^{\prime \prime}} \delta_{l^{\prime} l^{\prime \prime \prime}} .
$$

In (64) the ranges for $s$ and $s^{\prime}$ are $\max \left(0, L-l^{\prime}\right) \leqq s \leqq \min (L, l)$ and $\max \left(0, L-l^{\prime \prime \prime}\right) \leqq s^{\prime} \leqq \min \left(L, l^{\prime \prime}\right)$. By using (61) with (11) we have for $G_{L}^{s, s^{\prime}}(t)$ the formula

$$
\begin{aligned}
G_{L}^{s, s^{\prime}}(t)= & \frac{\left[(2(L-s)+1) !(2 s+1) !\left(2\left(L-s^{\prime}\right)+1\right) !\left(2 s^{\prime}+1\right) !\right]^{1 / 2}}{(L-s) !\left(L-s^{\prime}\right) !(2 L) !} \\
& \cdot \sum_{k=-\min \left(s, s^{\prime}\right)}^{\min \left(s, s^{\prime}\right)} \frac{(L-k) !(L+k) !}{(s+k) !\left(s^{\prime}+k\right) !} P_{s}^{k}(t) P_{s^{\prime}}^{k}(t) .
\end{aligned}
$$


From (65) we see that $G_{L}^{s, s^{\prime}}(t)$ is a polynomial of $t$ with degree $s+s^{\prime}$.

ii) $l+l^{\prime}-L=$ odd

We substitute $\left(2^{\prime}\right)$ into (62) and defining

$$
G_{L}^{s, s^{\prime}}(t) \equiv(4 \pi)^{2} \sum_{M} B_{L, M}^{s, L-s+1}\left(\Omega, \Omega^{\prime}\right) B_{L, M}^{s^{\prime}, L-s^{\prime}+1^{*}}\left(\Omega, \Omega^{\prime}\right),
$$

we have again (64) with a slight changes for the ranges over $s$ and $s^{\prime}$; $\max \left(1, L-l^{\prime}\right) \leqq s \leqq \min (L, l)$ and $\max \left(1, L-l^{\prime \prime}\right) \leqq s^{\prime} \leqq \min \left(L, l^{\prime}\right)$. In this case $G_{L}^{s, s^{\prime}}(t)$ is written as

$$
\begin{aligned}
G_{L^{\prime}}^{s, s^{\prime}}(t)= & \frac{[(2(L-s)+3)(2(L-s)+1) !(2 s+1)(2 s-1) !}{(L-s) !\left(L-s^{\prime}\right) !} \\
& \frac{\left.\cdot\left(2\left(L-s^{\prime}\right)+3\right)\left(2\left(L-s^{\prime}\right)+1\right) !\left(2 s^{\prime}+1\right)\left(2 s^{\prime}-1\right) !\right]^{1 / 2}}{(L+1)(2 L) !} \\
& \cdot \sum_{k=-\min \left(s, s^{\prime}\right)}^{\max \left(s, s^{\prime}\right)} \frac{2 k^{2}(L-k) !(L+k) !}{(s+k) !\left(s^{\prime}+k\right) !} P_{s}^{k}(t) P_{s^{\prime}}^{k}(t) .
\end{aligned}
$$

The orthogonality relations for $F_{L ; s}^{l, l^{\prime}}(t)$ take slightly simpler forms if we define new functions $H_{L ; k}^{l, l^{\prime}}(t)$ by

$H_{L ; k}^{l, l^{\prime}}(t)=\left[\frac{(L-k) !(L+k) !}{(2 L) !}\right]^{1 / 2}$

- $\sum_{s} F_{L ; s}^{l, l^{\prime}}(t) \frac{[(2(L-s)+1) !(2 s+1) !]^{1 / 2}}{(L-s) !(s+k) !} P_{s}^{k}(t), \quad$ for $\quad l+l^{\prime}-L=$ even, and

$$
\begin{aligned}
& H_{L ; k}^{l, l^{\prime}}(t)=\left[\frac{2(L-k) !(L+k) !}{(L+1)(2 L) !}\right]^{1 / 2} \\
& \cdot k \sum_{s} \frac{[(2(L-s)+3)(2(L-s)+1) !(2 s+1)(2 s-1) !]^{1 / 2}}{(L-s) !(s+k) !}
\end{aligned}
$$

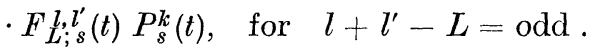

The orthogonality relations for $H_{L ; k}^{l, l^{\prime}}(t)$ are given by

$$
\sum_{k=-n}^{n} \int_{-1}^{1} d t H_{L ; k}^{l, l^{\prime}}(t) H_{L ; l^{\prime \prime}}^{l^{\prime \prime}, l^{\prime \prime \prime}}(t)=2(2 L+1) \delta_{l l^{\prime \prime}} \delta_{l^{\prime} l^{\prime \prime \prime}},
$$

with $n=\min \left(L, l, l^{\prime \prime}\right)$ for both cases.

Substituting $(18)\left(\left(18^{\prime}\right)\right)$ into $(68)\left(\left(68^{\prime}\right)\right)$, we obtain for both cases $H_{L ; k}^{l, l^{\prime}}(t)=(-)^{l-k}\left[\frac{(2 l+1)(2 L+1)(l-k) !}{(l+k) !}\right]^{1 / 2} C\left(L l l^{\prime} ; k,-k\right) P_{l}^{k}(t)$,

where we have used

and

$$
P_{s}^{k}(t)=\frac{(-) k\left(1-t^{2}\right)^{k / 2}(2 k) !}{k ! 2^{k}} C_{s-k}^{\frac{1}{2}+k}(t)
$$

$$
\sum_{s=k}^{m} \frac{2 k(m+s-1) !}{(s+k) !} C_{m-s}^{\frac{1}{2}-m}(t) C_{s-k}^{\frac{1}{2}+k}(t)=\delta_{m k},
$$

the proof of which is given in the Appendix A. 
Incidentally it should be noted that if one equate $(68)\left(\left(68^{\prime}\right)\right)$ to $(70)$ one obtains the relations between $F F_{L ; s}^{l, l^{\prime}}(t)$. These relations were also obtained by Joos [1] by a different method.

Finally we note the orthogonality relations for $B_{L, M}^{l, l^{\prime}}\left(\Omega, \Omega^{\prime}\right)$ for the sake of completeness;

$\int B_{L, M}^{l, l^{\prime *}}\left(\Omega, \Omega^{\prime}\right) B_{L^{\prime}, M^{\prime}}^{l^{\prime \prime}, l^{\prime \prime \prime}}\left(\Omega, \Omega^{\prime}\right) d \Omega d \Omega^{\prime}=\delta_{l l^{\prime \prime}} \delta_{l^{\prime} l^{\prime \prime}} \delta_{L L^{\prime}} \delta_{M M^{\prime}} \delta\left(l l^{\prime} L\right)$,

where $\delta\left(l l^{\prime} L\right)=1$ for $\left|l-l^{\prime}\right| \leqq L \leqq l+l^{\prime}$,

$=0$ otherwise.

Acknowledgement. The author wishes to express his sincere thanks to Dr. M. Yamamura and Mr. N. Setō for their helpful discussions.

\section{Appendix A. The Addition Formulas for the Bi-Linear Products of the Gegenbauer Polynomials}

We shall prove various addition formulas for the bi-linear products of the Gegenbauer polynomials which are used in the text. At first we list various recurrence relations for $C_{n}^{v}(t)$ which are used for the later discussions. Starting from the standard ones [3]

we get

$$
\begin{aligned}
(n+2 v) C_{n}^{v} & =2 v\left(C_{n}^{v+1}-t C_{n-1}^{v+1}\right), \\
n C_{n}^{v} & =2 v\left(t C_{n-1}^{v+1}-C_{n-2}^{v+1}\right),
\end{aligned}
$$

and

$$
C_{n}^{v}=C_{n}^{v+1}-2 t C_{n-1}^{v+1}+C_{n-2}^{v+1}
$$

$$
(n+v) C_{n}^{v}=v\left(C_{n}^{v+1}-C_{n-2}^{v+1}\right) .
$$

Multiplying $k$ on both sides of (A.4) and subtracting from (A.2) we have

$$
(n+2 v-k) C_{n}^{v}=(2 v-k) C_{n}^{v+1}-2(v-k) t C_{n-1}^{v+1}-k C_{n-2}^{v+1} .
$$

From (A.2) and (A.3) we also have

$$
n C_{n}^{v+1}=2(n+v) t C_{n-1}^{v+1}-(n+2 v) C_{n-2}^{v+1} .
$$

We follow the convention (a) in $\$ 3$ and prove

$$
\frac{(m+l) !}{(p+l) !(m-p) !} C_{l-p}^{\frac{1}{2}+p}(t)=\sum_{s} \frac{(-)^{s-p}(m+p) !}{(s+p) !(m-s) !} C_{l-s}^{\frac{1}{2}+m}(t) C_{s-p}^{\frac{1}{2}+p}(t) .
$$

According to the convention (a) the sum over $s$ on the right-hand-side

(r.h.s.) of (A.7) means $\sum_{s=p}^{\min (m, l)}$, hence for $p>\min .(m, l),($ A.7) is a trivial identity $0=0$. Assuming $p \leqq l$ we prove (A.7) by the mathematical

\footnotetext{
${ }^{6}$ (A.3) is derived for $v \neq 0$, but it is also valid for $v=0$.
} 
induction about $m$. For $m=p\left(\right.$ A.7) holds since r.h.s. $=1$.h.s. $=C_{l-p}^{\frac{1}{2}+p}(t)$. Hence we assume that (A.7) is valid for certain $m(>p)$ and prove that (A.7) holds also for $m+1$.

For $m \rightarrow m+1$

$$
\begin{aligned}
& \text { 1.h.s. of }(\mathrm{A} .7)=\frac{m+l+1}{m+1-p} \cdot \frac{(m+l) !}{(p+l) !(m-p) !} C_{l-p}^{\frac{1}{2}+p}(t) \\
= & \frac{m+l+1}{m+1-p} \sum_{s=p}^{m} \frac{(m+p) !}{(s+p) !(m-s) !}(-)^{s-p} C_{l-s}^{\frac{1}{2}+m}(t) C_{s-p}^{\frac{1}{2}+p}(t) .
\end{aligned}
$$

Substituting (A.5) with $v=1 / 2+m, n=l-s$ and $k=m-s$ in the above we have

$$
\begin{aligned}
& \text { l.h.s. of }(\mathrm{A} .7)=\frac{1}{m+1-p} \sum_{s=p}^{m} \frac{(-)^{s-p}(m+p) !}{(s+p) !(m-s) !}\left[(m+s+1) C_{l-s}^{m+3 / 2}\right. \\
- & \left.(2 s+1) t C_{l-s-1}^{m+3 / 2}-(m-s) C_{l-s-2}^{m+3 / 2}\right] C_{s-p}^{\frac{1}{2}+p} \\
= & \frac{(m+p) !}{(m+1-p)} \sum_{s=p}^{m+1} \frac{(-)^{s-p} C_{l-s}^{m+3}}{(s+p) !(m-s+1) !}\left[(m-s+1)(m+s+1) C_{s-p}^{\frac{1}{2}+p}\right. \\
+ & \left.(s+p)(2 s-1) t C_{s-p-1}^{\frac{1}{2}+p}-(s+p)(s+p-1) C_{s-p-2}^{\frac{1}{2}+p}\right] .
\end{aligned}
$$

We use (A.6) with $v=p-1 / 2$ and $n=s-p$ in the last factor of the above equation and get an equation $m \rightarrow m+1$ in (A.7). Hence (A.7) is valid under the convention (a).

In an exactly similar manner as above we can prove that

$$
\frac{(l-p) !}{(l-m) !(m-p) !} C_{l-p}^{\frac{1}{2}+p}(t)=\sum_{s} \frac{(-)^{m-s}(m+p) !}{(s+p) !(m-s) !} C_{l-2 m+s}^{\frac{1}{2}+m}(t) C_{s-p}^{\frac{1}{2}+p}(t) .
$$

Next we consider the analytic continuation in $m$ in (A.7). Eq. (A.7) is proved for integral points $m=p, \mathrm{p}+1, p+2, \ldots$. However, the $m$-dependence of the l.h.s. of (A.7) is given by $\Gamma(m+l+1) / \Gamma(m-p+1)$ and that of r.h.s. is inferred from

$$
C_{l-s}^{\frac{1}{2}+m}(t)=\sum_{\substack{\sigma, \tau=0 \\ \sigma+\tau=l-s}}^{l-s} \frac{\Gamma\left(\frac{1}{2}+m+\sigma\right) \Gamma\left(\frac{1}{2}+m+\tau\right)}{\tau ! \sigma ! \Gamma\left(\frac{1}{2}+m\right) \Gamma\left(\frac{1}{2}+m\right)} \cos (\sigma-\tau) \Theta
$$

apart from $\Gamma(m+p+1) / \Gamma(m-s+1)$. Therefore the asymptotic dependence on $m$ allows us the use of the Carlson's theorem [7], and (A.7) is valid for $\operatorname{Re} m \geqq p$. However, the analytic domain where (A.7) holds is further enlarged to the whole $m$-plane, because the both sides of (A.7) are simply rational functions of $\Gamma$-functions. By using

$$
\begin{aligned}
\Gamma(m-p+1) / & \Gamma(m-s+1) \\
& =\Gamma(s-m) \sin \pi(m-s+1) / \Gamma(p-m) \sin \pi(m-p+1),
\end{aligned}
$$

3 Commun. math. Phys., Vol. 9 
we put $m=-l$ in (A.7), getting

$$
C_{l-p}^{\frac{1}{2}+p}(t) /(p+l) \Gamma(p-l+1)=\sum_{s} \frac{(s+l-1) !}{(s+p) !} C_{l-s}^{\frac{1}{2}-l}(t) C_{s-p}^{\frac{1}{2}+p}(t) .
$$

The 1.h.s. of the above equation is zero for $l-p= \pm 1, \pm 2, \pm 3, \ldots$ and $1 / 2 l$ for $l-p=0$, hence we have

$$
\sum_{s=p}^{l} \frac{2 l(l+s-1) !}{(s+p) !} C_{l-s}^{\frac{1}{2}-l}(t) C_{s-p}^{\frac{1}{2}+p}(t)=\delta_{l p} .
$$

(A.10) can also be proved in an elementary way by using various recurrence relations and a relation

$$
\sum_{k=0}^{l} C_{k}^{\lambda}(t) C_{l-k}^{\mu}(t)=C_{l}^{\lambda+\mu}(t)
$$

which is a direct consequence from the generating function of the Gegenbauer polynomials, (25). However, the procedure is much tedious.

In order to prove (14) in $\S 2$, we define $f_{k s}(t)$ and $h_{k s}(t)$ according to (13) and (15). Further we define matrices

$$
(F)_{k s}=f_{k s}(t), \quad(H)_{k s}=h_{k s}(t)(-)^{k-s} .
$$

Then (A.10) is rewritten simply as

$$
H F=1 \text {. }
$$

Hence, we have

$$
F H=1 \text {, }
$$

which can be rewritten

$$
\begin{aligned}
\sum_{s=p}^{k} f_{k s}(t)(-)^{s-p} h_{s p}(t) & =\sum_{s=p}^{k} \frac{(2 s)(s+p-1) !}{(k+s) !} C_{k-s}^{\frac{1}{2}+s}(t) C_{s-p}^{\frac{1}{2}-s}(t) \\
& =\delta_{k p} .
\end{aligned}
$$

(A.15) is used in $\S 2$.

By using (A.15) we have from (A.7) and (A.8)

$\sum_{k} \frac{(2 k)(-)^{k-s}(k+s-1) !}{(l+k) !(m-k) !(m+k) !} C_{l-k}^{\frac{1}{2}+k}(t) C_{k-s}^{\frac{1}{2}-k}(t)=\frac{C_{l-s}^{\frac{1}{2}+m}(t)}{(m+l) !(m-s) !}$,

and

$\sum_{k} \frac{(2 k)(l-k) !(k+s-1) !}{(m-k) !(m+k) !} C_{l-k}^{\frac{1}{2}+k}(t) C_{k-s}^{\frac{1}{2}-k}(t)=\frac{(-)^{m-s}(l-m) !}{(m-s) !} C_{l-2 m+s}^{\frac{1}{2}+m}(t)$

$$
\text { for } l \geqq m \text {. }
$$

(A.17) is used in $\S 3$.

Incidentaly it should be noted that (A.16) and (A.17) furnish the direct proof of the symmetry properties of $F_{L ; s}^{l, l^{\prime}}(t)$. For that purpose we insert into $(18)\left(\left(18^{\prime}\right)\right)$ the expression for the CGC given by WIGNER [6] 
instead of (23),

$$
\begin{aligned}
C\left(L l l^{\prime} ; k,-k\right)= & {\left[\frac{\left(2 l^{\prime}+1\right)\left(l+l^{\prime}-L\right) !\left(l^{\prime}+L-l\right) !}{\left(l+l^{\prime}+L+1\right) !\left(l^{\prime}\right) !\left(l^{\prime}\right) !}\right.} \\
& \left.\frac{\cdot\left(L+l-l^{\prime}\right) !(L-k) !(L+k) !}{\cdot(l-k) !(l+k) !}\right]^{1 / 2} \\
& \cdot \sum_{v} \frac{(-)^{v}(l+L-v) !\left(l^{\prime}+v\right) !}{v !\left(L-l^{\prime}+l-v\right) !(L-k-v) !\left(v+l^{\prime}-l+k\right) !} .
\end{aligned}
$$

We repeat the similar calculations as those in $\S 3$, and thereby use (A.16) instead of (A.17) and finally obtain $(44)((60))$ directly. In the text we have obtained (44) $((60))$ from $(43)((59))$ by using the symmetry properties. Therefore we have proved the symmetry properties of $F_{L ; s}^{l, l^{\prime}}(t)$ defined by $(18)\left(\left(18^{\prime}\right)\right)$ only by using the properties of CGC and the Gegenbauer polynomials.

\section{Appendix B}

In this appendix we shall prove various formulas which are used in the text.

Proof of Eqs. (30) and (50)

From the defining Eq. (29) of $g_{m ; k}^{a}$ we have a recurrence relation

$$
\begin{gathered}
g_{m: k}^{a}=-2 g_{m-1 ; k}^{a}+(2 a+1)(2 a+2) g_{m-1 ; k}^{a+1}-g_{m-2 ; k}^{a} \\
(m=2,3,4, \ldots) .
\end{gathered}
$$

Eq. (30) satisfies (B.1) as can easily be verified, hence (30) is proved by the mathematical induction about $m$. Similarly, from the defining equation of $h_{m ; k}^{a+1}(t)$ we have a recurrence relation

$$
h_{m ; k}^{a+1}=-2 h_{m-1 ; k}^{a+1}+(2 a+2)(2 a+3) h_{m-1 ; k}^{a+2}-h_{m-2 ; k}^{a+1}
$$

Eq. (50) satisfies (B.2) and is proved in a similar manner.

Proofs of Eqs. (34), (35) and (53)

According to the convention (a) in $\S 3$, the defining Eq. (32) of $\varphi_{b, c}^{o}$ is symmetric between $b$ and $c$, hence we prove (34) in the case $c \geqq b$. Eq. (34) is valid for $b=\varrho$. (We assume $\varrho \geqq 1$ ). From Eq. (32) we have a recurrence relation

$$
\left(b^{2}-\varrho^{2}\right) \varphi_{b, c}^{\varrho}=\varphi_{b-1, c}^{\varrho}+\varphi_{b, c}^{o+1} .
$$

After some manipulations it can easily be verified that (34) satisfies (B.3). Hence, by the mathematical induction about $b-\varrho$ we have proved (34).

For the proof of (35) we can also assume $c \geqq b$. From the defining Eq. (33) for $\psi_{b, c}$, and (B.3) with $\varrho=0$ we have a recurrence relation

$$
b^{2} \psi_{b, c}=\psi_{b-1, c}+\frac{(2(b+c)-3) !}{(b+c) !(2 b-1) !(2 c-1) !(b+c-2) !},
$$


where we have used (34) with $\varrho=1$. For $b=0,(35)$ is valid since (33) leads to $\psi_{0, c}=(c !)^{-2}$. As can easily be verified, (35) satisfies (B.4), hence the mathematical induction about $b$ guarantees the validity of (35).

To prove (53) we get from (52) a recurrence relation

$$
(b-\varrho)(b+\varrho+1) \chi_{b, c}^{\varrho}=\chi_{b-1, c}^{\varrho}+\chi_{b, c}^{\varrho+1} .
$$

(53) satisfies (B.5) and its validity is proved in a similar way as above.

Proof of Eqs. (42) and (58)

Putting $b-v=\varrho$ and $a+b=L$ in the defining Eq. (41), we write $\bar{\phi}_{d, L, L-s}^{\varrho} \equiv \phi_{c, L, s}^{\varrho}$ with $d \equiv a+b+c+1=L+c+1$. Then we have $\bar{\phi}_{d, L, s}^{\varrho}=\sum_{\sigma=0}^{\varrho} \frac{(-)^{\sigma}(L-\varrho+\sigma) !(d-s-\sigma) !(2(d-L-1+\varrho-\sigma)) !}{\sigma !(\varrho-\sigma) !(s-\varrho+\sigma) !(2(d-s-\sigma)) !(d-L-1+\varrho-\sigma) !}$.

From (B.6) we have a recurrence relation

$$
s \bar{\phi}_{d, L+1, s}^{o+1}=(L+1) \bar{\phi}_{\bar{d}-1, L, s-1}^{o+1}+(L-s+1) \bar{\phi}_{d-1, L-1, s-1}^{\varrho},
$$

For $s=0$ we get from (B.6)

$$
\bar{\phi}_{d, L, s=0}^{\varrho}=\frac{(-)^{\varrho} L !(d-\varrho) !(2(d-L-1)) !}{\varrho !(2(d-\varrho)) !(d-L-1) !}
$$

and putting $s=1$ in (B.7) we get $\bar{\phi}_{d, L, s=1}^{o}$. Therefore successive use of (B.7) gives for general $s$

$$
\bar{\phi}_{d, L, s}^{\varrho}=\frac{(-)^{\varrho}(d-s) !(d-\varrho) !(L-s) !(L-\varrho) !(2 d) !(2(d-L-1)) !}{(2(d-s)) !(2(d-\varrho)) ! s ! \varrho ! d !(d-L-1) !(L-s-\varrho) !},
$$

which satisfies (B.7) as can easily be verified. Rewriting (B.8) in terms of the original variables we get (42) in the text.

Similarly putting $b-v=\varrho$ and $a+b+1=L$ in the defining Eq. (57) we write $\bar{\psi}_{d, L, L-s}^{\varrho} \equiv \psi_{c, L, s}^{\varrho}$ with $d=a+b+c+2=L+c+1$. Then we have

$\bar{\psi}_{d, L, s}^{\varrho}=\sum_{\sigma=0}^{\varrho} \frac{(-)^{\sigma}(L-\varrho+\sigma-1) !(d-s-\sigma) !(2(d-L+\varrho-\sigma)-1) !}{\sigma !(\varrho-\sigma) !(s-\varrho+\sigma) !(2(d-s-\sigma)) !(d-L+\varrho-\sigma-1) !}$.

From (B.9) we have a recurrence relation

$$
s \bar{\psi}_{d+1, L+1, s}^{\varrho+1}=L \bar{\psi}_{d, L, s-1}^{\varrho+1}+(L-s) \bar{\psi}_{d, L-1, s-1}^{\varrho} .
$$

Successive use of (B.10) gives for general $s$

$$
\bar{\psi}_{d, L, s}^{\varrho}=\frac{(-)^{\varrho}(L-\varrho-1) !(2 d) !(2(d-L)-1) !(d-s) !(d-\varrho) !(L-s-1) !}{\varrho ! s ! d !(2(d-s)) !(2(d-\varrho)) !(d-L-1) !(L-s-\varrho-1) !} .
$$

Rewriting (B.11) in terms of the original variables we get (58) in the text. 


\title{
References
}

1. Joos, H.: Fortschr. Physik 10, 65 (1962).

2. Rose, M. E.: Elementary theory of angular momentum, Chap. IV, V. New York: John Wiley and Sons 1957.

3. Erdelyi, A.: Bateman manuscript project. Higher transcendental functions. Vol. I, p. 175. London, Toronto, New York: McGraw-Hill Book Company, Inc. 1953.

4. RACAH, G.: Phys. Rev. 62, 438 (1942).

5. Domokos, G.: Phys. Rev. 159, 1387 (1967); Freedmax, D. Z., and Wang, J. M.: Phys. Rev. 160, 1560 (1967).

6. Wigner, E. P.: Gruppentheorie und ihre Anwendung auf die Quantenmechanik der Atomspektren. Braunschweig: Friedrich Vieweg und Sohn 1931.

7. TItchmaRsh, E. C.: Theory of functions, Section 5.81. New York: Oxford Univ. Press. 1939.

\author{
Yasuo Munakata \\ Department of Physics \\ Kyoto University \\ Kyoto, Japan
}

